\title{
Platelet Rich Plasma: Current Indications and Use In Orthopaedic Care
}

\author{
Author: \\ Richard Judd Robins, MD, \\ Assistant Professor of Surgery \\ Uniformed Services University of \\ the Health Sciences \\ Dept. of Orthopaedics; \\ Sports Medicine Service \\ U.S. Air Force Academy \\ E-mail: RobinsRJ@Hotmail.com
}

\begin{abstract}
"Platelet Rich Plasma" (PRP) is a ubiquitous term for a type of therapy that applies autologous platelets collected from whole blood and then concentrated via centrifuge that are re-applied to injured musculoskeletal tissue. The intent of most PRP therapies is to locally create or support a "healing response," that instigates earlier healing, or to initiate a response in chronic degenerative and painful tissue. Complicating research efforts are the high number of different types and methods of PRP therapies, and the multiple anatomic and tissue sites of treatment that may potentially benefit from PRP. One of the greatest challenges in the development of PRP therapy have been translating promising laboratory and animal study results to clinical studies' outcomes. While enthusiasm for PRP therapy has often exceeded the evidence to support therapeutic benefits, recent studies and experience does support the use of specific types of PRP therapy in specific degenerative and injury patterns.
\end{abstract}

Keywords: Platelet Rich Plasma; Tendinosis; Osteoarthritis; Injection Therapy 
Medical Research Archives, Vol. 5, Issue 6, June 2017

Platelet Rich Plasma: Current Indications and Use in Orthopaedic Care

\section{Introduction}

Recovery from musculoskeletal injuries at times can be a slow and incomplete process leading to prolonged time away from work, sport, and loss of functional capacity. The repair process after injury starts with the inflammatory process, and is regulated by complex interactions of multiple growth factors to help restore damaged tissue. Over the last decade, efforts to improve or expedite healing injuries in the musculoskeletal system have focused on applying these growth factors. PRP has gained traction in research and treatment efforts as PRP provides a relatively simple, low cost, and fairly easy mode to acquire these growth factors in what is assumed to be physiologically balanced concentrations that can then be applied to damaged tissue.(Kon, Filardo, Di Martino, \& Marcacci, 2011) PRP is obtained from a patient's own whole blood (typically 15-60cc) that is centrifuged once or twice to separate out platelets with (double spin) or without (single spin) white blood cells, with concentrations anywhere from twice to nine times normal concentrations. It is most often mixed with an exogenous activator of platelets (ex: thrombin, calcium chloride, calcium citrate) which causes release of alpha granules from the platelets containing multiple cytokines and growth factors. The activated platelet concentrate is then injected into a damaged musculoskeletal tissue site (tendon, muscle, joint/cartilage, etc.) to either activate or enhance a "healing response." In more acute settings, endogenous activation relies on the platelet concentrate being activated when coming in contact with damaged collagen or other musculoskeletal tissue. Protocols call for either 1 to 4 injections spread out at least 4 to 7 days based upon evidence that release of cytokines and growth factors primarily occur in the first hours after injection, while platelet viability and cytokine release continues up to seven days after activation.(Marx, 2004)

Categories of PRP application fall into the 4 areas: chronic tendinosis, acute ligament injury, muscle injury, and intraoperative tissue repair augmentation.(Hsu et al., 2013) Enthusiasm for clinical use and application of PRP has often outpaced solid evidence to support its use.(Sampson, Gerhardt, \& Mandelbaum, 2008) The typical pattern in PRP research has shown very promising results in laboratory and animal studies, but translation of PRP therapy to clinical trials has been at times disappointing.(Robins, 2014) However, recent advances in specific areas of treatment with specific types of PRP therapy have demonstrated good evidence to support use in clinical settings.(Fitzpatrick, Bulsara, \& Zheng, 2017; Gosens, Peerbooms, van Laar, \& den Oudsten, 2011; Hsu et al., 2013; Meheux, McCulloch, Lintner, Varner, \& Harris, 2016)

\section{Basic Science of PRP}

PRP falls under a class of therapeutic approaches termed "biologics," which refers to using natural products to augment the physiological process of healing. At times, PRP may be classified under the umbrella of "prolotherapy" or "regenerative medicine," but PRP does not precisely meet the definition or the intent of these therapeutic philosophies. From a regulatory standpoint, the FDA defines "biologics" or biologic therapy as requiring minimal manipulation of an autologous source of cellular treatment, and whose efficacy does not relate to the metabolic activity of the cells being applied for therapy. Biologic treatment currently falls into 3 classes of therapy: growth factors (PRP), cells (ex: stem cells), and tissue patches for surgical augmentation.(Williams, Moran, Bradley, N, \& Dines, 2015) 
PRP therapy is based on the premise that it benefits musculoskeletal tissue healing due to the high concentration of cytokines and growth factors that normally are involved in the initial and follow-on stages of the inflammatory process at recruiting and stimulating cell proliferation as well as producing extra-cellular matrix proteins.(Anitua et al., 2006; Williams et al., 2015) A list of these growth factors and cytokines are listed in Table 1. While the theoretical advantage of using PRP administers these growth factors in physiological concentrations relative to each other, the reality is that these concentrations vary depending of the method of PRP preparations, amount of whole blood used, type of platelet activation, and the final total volume of PRP injected.(Fitzpatrick, Bulsara, McCrory, Richardson, \& Zheng, 2017) In addition, the concentration of platelets and associated cytokines varies between patients, and even within the same patient at different points in time.(Creaney \& Hamilton, 2008; Mazzocca et al., 2012) In other words, a patient receiving a series of treatment of 3 PRP injections one week apart, even when standardizing for type of PRP preparation, activation, and volume administered, will receive different concentrations of platelets in each of the PRP injections due to the variability of available platelets and leukocytes in the whole blood obtained from the patient at the time of collection.

\subsection{Challenges in PRP Research}

Many factors make interpreting and applying PRP research results challenging to clinical application. Two of these biggest factors include the plethora of different PRP preparations available for use, and the challenge of translating the success found in laboratory and animal research to clinical outcomes.

\subsubsection{PRP Preparation Variability}

PRP encompasses many variations of preparation methods, but can be best divided into plasma-based and "buffy-coat" based preparations. Plasma-based preparations are a result of a "single-spin" centrifuge process, which separates out and concentrates red blood cells, the "buffy coat" made up of white-blood cells, and plasma, which contains platelets concentrated on average between 2-5 times that of whole blood (Figure 1). If the preparation undergoes a second centrifuge spin (double spin), this serves to concentrate the platelets even further, resulting in a "platelet-rich" and a "platelet-poor" plasma, but also include leukocytes in the platelet rich portion of the plasma. This results in different effects from the prepared PRP, with plasma based, single-spin PRP preparations demonstrating anabolic and cartilage extra-cellular-matrix stabilization effects, while white blood cell-containing double-spin PRP preparations create a stronger inflammatory and catabolic response.(Braun, Kim, Chu, \& Dragoo, 2014) As a result, the indications of treatment may dictate that different preparations may provide a better response based upon the tissue environment and pathology being treated. 
Medical Research Archives, Vol. 5, Issue 6, June 2017

Platelet Rich Plasma: Current Indications and Use in Orthopaedic Care

Table 1

Growth Factors and Active Biomolecules in Platelet Rich Plasma (PRP)

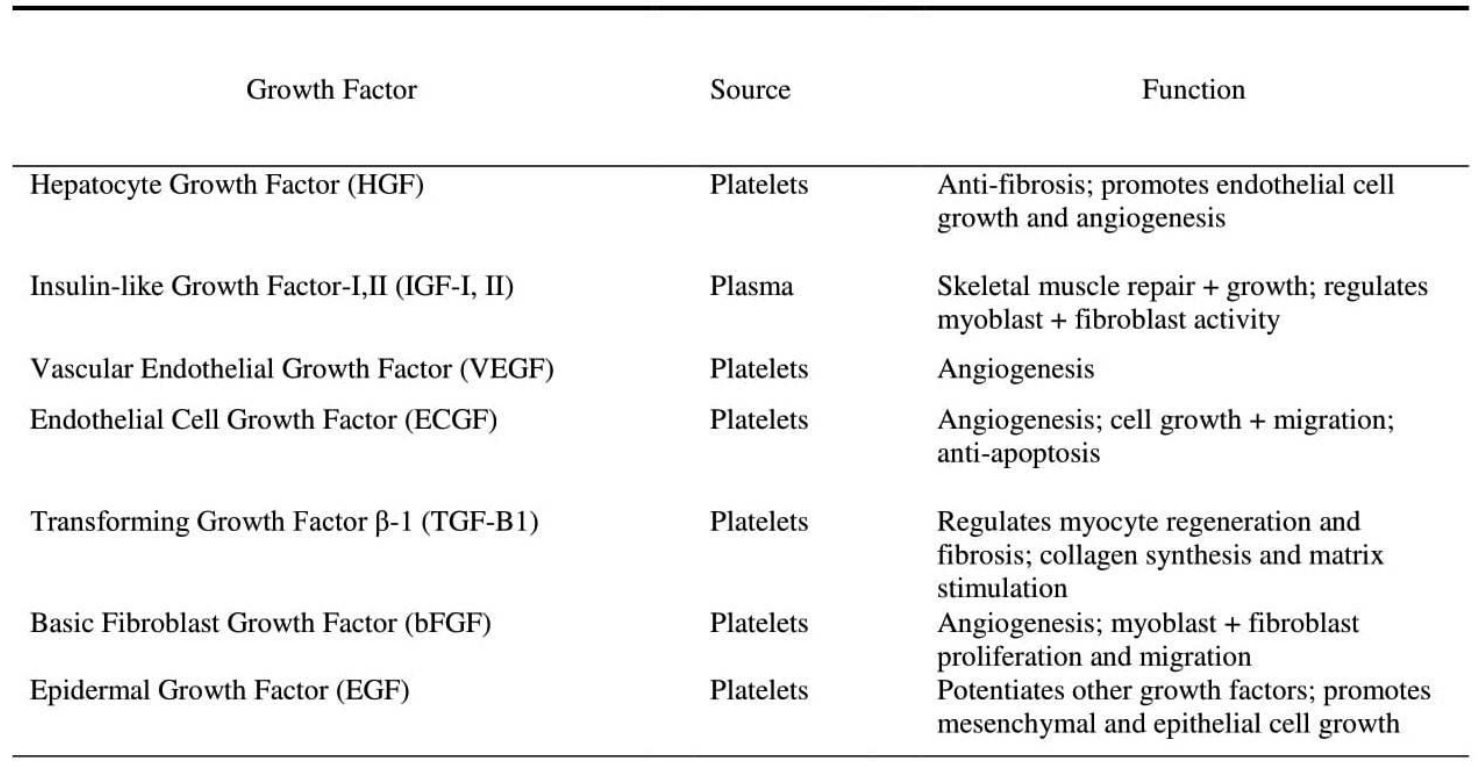

Bioactive Molecule

Source

Function

\begin{tabular}{|c|c|c|}
\hline Fibrinogen & Platelets & Fibrin clot formation \\
\hline Fibronectin & Platelets & $\begin{array}{l}\text { Affects cell growth, migration, } \\
\text { differentiation, and adhesion }\end{array}$ \\
\hline Vitronectin & Platelets & cell adhesion \\
\hline Thrombospondin-1 & Platelets & Inhibits angiogenesis \\
\hline Factor V & Platelets & Thrombin activation + clot formation \\
\hline Factor XI & Platelets & Thrombin activation + clot formation \\
\hline Protein S & Platelets & Thrombin activation + clot formation \\
\hline Antithrombin & Platelets & Thrombin activation + clot formation \\
\hline Plasminogen & Platelets & Fibrinolysis \\
\hline Plasminogen activator inhibitor & Platelets & Regulates Plasmin production \\
\hline$\alpha$-2 Antiplasmin & Platelets & Inactivates Plasmin \\
\hline Tissue Inhibitor of Metalloprotease-4 (TIMP-4) & Platelets & Regulates Matrix Degradation \\
\hline Metalloprotease- 4 & Platelets & Matrix Degradation \\
\hline$\alpha 1$-antitrypsin & Platelets & Inhibits proteases \\
\hline Platelet factor 4 & Platelets & Inhibits angiogenesis \\
\hline$\beta$-Thromboglobulin & Platelets & $\begin{array}{l}\text { Inhibits angiogenesis; promotes } \\
\text { angiogenesis }\end{array}$ \\
\hline Endostatins & Platelets & $\begin{array}{l}\text { Inhibits angiogenesis + endothelial cell } \\
\text { migrations }\end{array}$ \\
\hline CD40 Ligand & Platelets & $\begin{array}{l}\text { Synthesizes interleukins and integrin; } \\
\text { promotes leukocyte migration and platelet } \\
\text { endothelial cell adhesion }\end{array}$ \\
\hline P-Selectin & Platelets & $\begin{array}{l}\text { Leukocyte migration and adhesion to } \\
\text { vascular cells }\end{array}$ \\
\hline
\end{tabular}


Medical Research Archives, Vol. 5, Issue 6, June 2017

Platelet Rich Plasma: Current Indications and Use in Orthopaedic Care

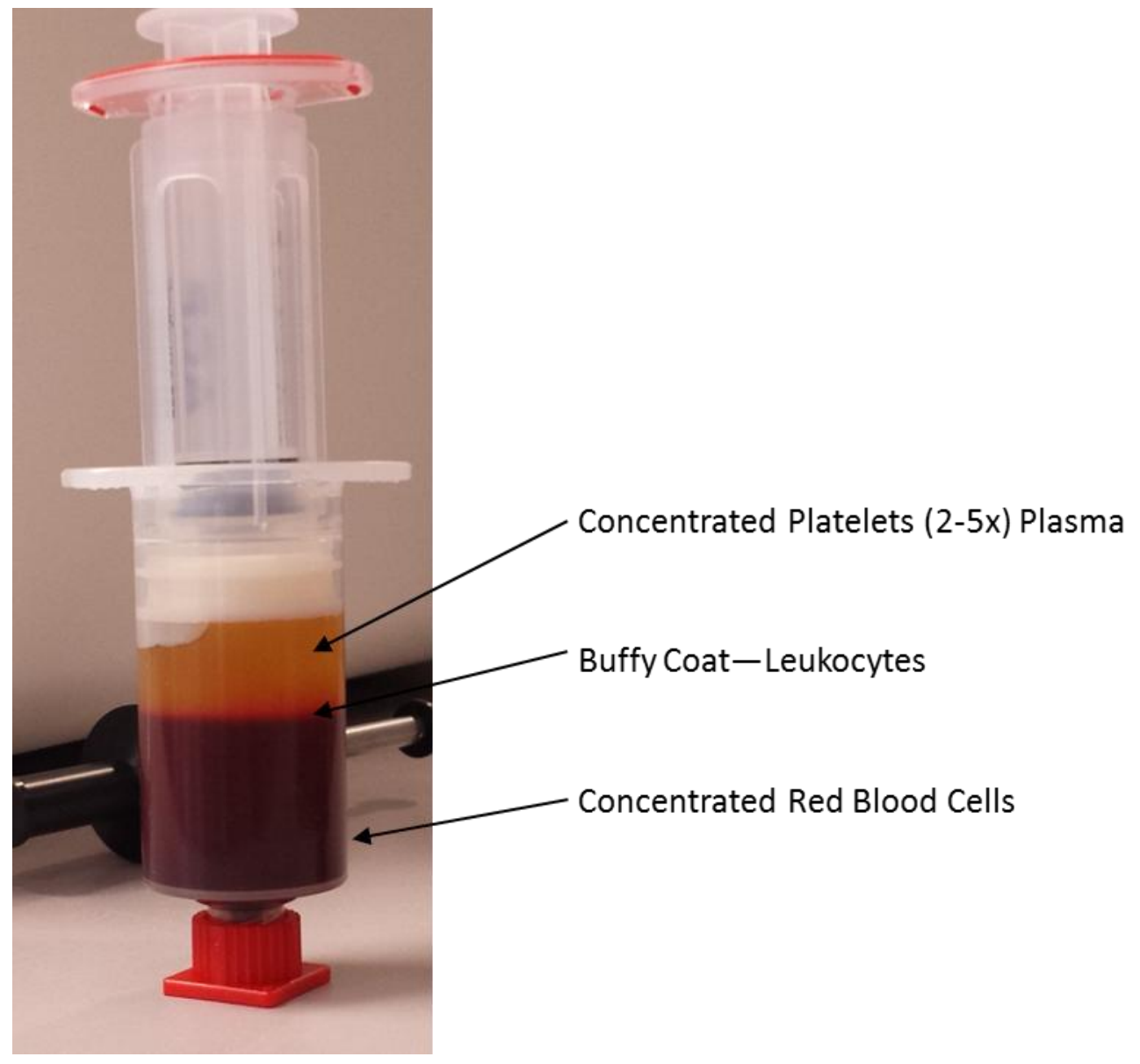

Figure 1. Sample of Single-Spin PRP Preparation Immediately After Centrifugation

\subsubsection{Challenges with Comparison Analysis}

When evaluating results of PRP, it is imperative to determine the type of preparation of PRP used. Mishra et al and DeLong and associates proposed classification systems be adopted when evaluating and comparing PRP that takes into account the concentration of platelets, the type of activation that occurs, and the absence or presence of white blood cells.(DeLong, Russell, \& Mazzocca, 2012; A. Mishra, Harmon, Woodall, \& Vieira, 2012) Using DeLong's "PAW" classification system, over 32 different types of PRP classifications are identified. DeLong's classification could be delineated even further by sub-classifying the different substrates used for exogenous activation (thrombin, calcium-chloride, calcium citrate, etc.) and endogenous preparations which could lead to approximately 160 different classifications of PRP. While multiple systematic reviews and metanalyses have been accomplished on PRP treatments, caution is recommended when interpreting the findings of all of these comparison studies, as all have used a collation of different PRP preparation studies to draw conclusions. Hall and colleagues noted that with the "qualitative and quantitative differences, reported evidence for clinical effectiveness of PRP cannot be generalized across preparation systems."(Hall, Band, Meislin, Jazrawi, \& Cardone, 2009) 
Medical Research Archives, Vol. 5, Issue 6, June 2017

Platelet Rich Plasma: Current Indications and Use in Orthopaedic Care

\section{Current Clinical Applications}

In addition to the multitude of PRP preparations, the different sites and types of injured tissue make comparison of PRP difficult. However, recent advances in specific pathologies has shown promising results, in particular for the treatment of mild to moderate degenerative joint disease of the knee, lateral epicondylitis, and patellar tendinitis. Other areas of treatment have included muscle belly injury, and surgical augmentation, in particular with rotator cuff tendon repair and anterior cruciate ligament reconstruction.

\subsection{Knee Osteoarthritis}

Use of PRP in preclinical data provided evidence that single spin, leukocyte poor preparations of PRP reduced inflammatory cytokines (in particular IL$1 b$ ), and helped to stabilize the extracellular matrix of damaged hyaline cartilage.(Durant et al., 2017; Filardo et al., 2015) In particular, PRP preparations with higher concentrations of white blood cells seemed to have a catabolic effect and increased the inflammatory response for intra-articular synovial cells verses plasma based PRP preparations that demonstrated an in-vitro anabolic, anti-inflammatory effect.(Braun et al., 2014; Filardo et al., 2012; Osterman et al., 2015; Riboh, Saltzman, Yanke, Fortier, \& Cole, 2016) Multiple lower level studies using plasma based PRP with low concentrations of white blood cells have been completed comparing this type of PRP to other forms of intra-articular injection therapy, which demonstrated positive effects at reducing pain and improving outcomes scores in degenerative joint disease of the knee.(Cerza et al., 2012; Filardo et al., 2013; Filardo et al., 2011) Smith performed a double blinded randomized control trial comparing autologous conditioned plasma (ACP — single spin, leukocyte poor, 2-5x platelet concentrated PRP) vs. saline injections in small but acceptably powered populations of patients with mild to moderate degenerative joint disease, and demonstrated statistically significant improvements in visual analog pain scores for 12 months after therapy, with 78\% improvement in WOMAC scores compared to $7 \%$ in the placebo control group.(Smith, 2016) Cole et al. performed a double blinded randomized control trial comparing hyaluronic acid vs the same PRP preparation used in the Smith study, which demonstrated improvement in pain symptoms in both groups, but significantly better outcomes scores at 24 weeks in the PRP study group.(Cole, Karas, Hussey, Pilz, \& Fortier, 2017) A recent metanalysis, while suffering from the limitations of comparative studies as noted above, evaluated PRP injections for knee degenerative disease and concluded PRP injections resulted in clinically significant improvements out to 12 months after therapy, with pain scores and clinical outcomes that were better than hyaluronic acid injections at 3-12 months.(Meheux et al., 2016) Indirect evidence demonstrated leukocyte poor PRP injections resulted in better clinical outcomes compared to hyaluronic acid than leukocyte rich PRP injections vs hyaluronic acid, but no direct comparison between the PRP preparations have been completed at this time. Another meta-analysis of only level-I studies demonstrated more pain relief and functional improvement in treating symptomatic knee osteoarthritis at 1 year post-injection when compared to hyaluronic acid injections.(Dai, Zhou, Zhang, \& Zhang, 2017) In summary, there is growing evidence to support the use of PRP injections for treatment of degenerative joint disease of the knee, with some basic science and clinical evidence favoring use of PRP preparations low in white blood cells.(Hunt, 2017) 
Medical Research Archives, Vol. 5, Issue 6, June 2017

Platelet Rich Plasma: Current Indications and Use in Orthopaedic Care

\subsection{Lateral Epicondylitis}

PRP therapy was initially thought to be a potentially beneficial treatment for chronic tendinopathy and tendinosis. To date, lateral epicondylitis has demonstrated the best response to PRP treatment of all chronic tendinopathy conditions. Unlike treatment of intra-articular and cartilage pathology, leukocyte-rich PRP therapies have demonstrated better pain scores and functional outcomes than leukocyte-poor PRP as well as have performed better than local anesthetics, corticosteroids, or dry needling.(Fitzpatrick, Bulsara, \& Zheng, 2017) Gosens and colleagues conducted a randomized control trial comparing corticosteroid injections to leukocyte-rich PRP, and noted $73 \%$ of patients improved in pain (VAS) and functional (DASH) outcomes scores versus approximately 50\% in the corticosteroid group over the $1^{\text {st }}$ year.(Peerbooms, Sluimer, Bruijn, \& Gosens, 2010) They also noted that the corticosteroid group initially had a quick response compared to the PRP group during the $1^{\text {st }} 6$ to 12 weeks, but the effects dropped off rapidly and returned to baseline, while the PRP group continued to demonstrate clinically significant improvements over the 2 years following treatment.(Gosens et al., 2011) These studies had similar findings when compared to Mishra and Pavelko's study which compared leukocyte-rich PRP to local anesthetic, and was able to find significant improvement in both VAS and functional outcomes out to 25.6 months.(A. Mishra \& Pavelko, 2006) A recent double blinded randomized control trail conducted at multiple centers compared leukocyte-rich PRP added to dry needling of patients who have failed prior conservative therapy. The study resulted in $84 \%$ of the PRP group having successful treatment vs. $68 \%$ in the needling-only group.(A. K. Mishra et al., 2014) A systematic review in 2013 noted that of 9 studies, only 2 randomized control trials showed no differences compared to corticosteroid injections, but that these studies were limited to 3 month follow up.(Ahmad et al., 2013) In contrast to this, a systematic review conducted in 2014 stated that no benefit was found from performing PRP for lateral epicondylitis.(de Vos, Windt, \& Weir, 2014) However, these findings have been disputed as using incorrect grading of the included studies, and drawing incorrect assumptions and conclusions based upon the studies included due to limited $(<24$ week) follow up.(Gosens \& Mishra, 2014) A recent randomized control trial compared leukocyte-poor PRP to corticosteroid injections, and found similar positive results in pain and DASH outcomes scores, favoring PRP treatment at 1 year.(Lebiedzinski et al., 2015) In conclusion, several small and large randomized trials have demonstrated better long-term outcomes with leukocyte-rich PRP injections over corticosteroid injections, with a more recent study demonstrating similar results with leukocyte-poor PRP over corticosteroids.

\subsection{Ulnar Collateral Ligament (UCL)}

Injuries to the UCL are common in overhead athletes, in particular to pitchers in baseball. While full UCL sprains require surgery and generally have good outcomes at returning athletes back to sports, recovery can take upwards of 12 months. In recent years, efforts have been made to evaluate if nonoperative interventions can be used to treat partial UCL tears over surgery. Podesta et al. evaluated grade I and II UCL sprains in 34 athletes treated with leukocyterich PRP with ultrasound guidance.(Podesta, Crow, Volkmer, Bert, \& Yocum, 2013) They noted by 6 months, $88 \%$ of athletes had returned to play at the same level without complaints, improvement on 
Medical Research Archives, Vol. 5, Issue 6, June 2017

Platelet Rich Plasma: Current Indications and Use in Orthopaedic Care

outcomes scores, and decrease in medial elbow joint space opening from $7 \mathrm{~mm}$ to $2.5 \mathrm{~mm}$. Dines and colleagues noted an earlier return to sport in 4 of 6 professional athletes with use of leukocyte-poor PRP for partial UCL injuries in baseball players, with return to competition between 5-24 weeks (average 12 weeks).(Dines et al., 2016) $72 \%$ of athletes had a good or excellent outcome. Though limited data is available, PRP therapy may be considered in partial UCL injuries as an option prior to surgical reconstruction.

\subsection{Patellar Tendinosis}

Initial clinical results demonstrated promise for using PRP in patellar tendinosis. A pilot study of 20 patients with refractory "jumper's knee" were treated with 3 injections using $5 \mathrm{~mL}$ of a double-spin, leukocyte-rich PRP (6x concentration of platelets) preparation activated with calcium chloride with 15 days in-between each injection.(Kon et al., 2009) At 6 months, statistically significant clinical improvements were noted in all factors including physical functional, with $80 \%$ of participants returned back to sport 4 months after therapy was administered. A follow-on comparative study evaluated patients refractory to established physical therapy treated with the same type of PRP as noted above with physical therapy only. The study demonstrated improved outcomes at 6 months in outcomes and pain scores in the study group, which represents a difficult patient cohort to successfully treat.(Filardo et al., 2010) A recent randomized control trial compared eccentric strengthening, dry needling, and one leukocyte-rich PRP to eccentric exercises and dry needling only.(Dragoo, Wasterlain, Braun, \& Nead, 2014) Results demonstrated PRP helped to improve initial recovery, but the results between the two groups equalized by 26 weeks. Gosens and colleagues noted clinically meaningful improvement after treatment with PRP in patients with patellar tendinopathy, but saw greater improvement in the patient cohort who had not had prior corticosteroid, ethoxysclerol, or surgery.(Gosens, Den Oudsten, Fievez, van 't Spijker, \& Fievez, 2012) Using 3 injections of leukocyte poor PRP (ACP) in a prospective study, $75 \%$ of patients had the ability to return to sports, with $57 \%$ of patients demonstrating complete resolution of tendon structure at 3 months from treatment. At 2 years, all patients were satisfied with the procedure with significant improvements in outcomes and pain scores.(Charousset, Zaoui, Bellaiche, \& Bouyer, 2014) A recent systematic review of 6 randomized control trials and 19 total studies demonstrated that patellar tendinopathy treated with PRP demonstrated benefits in the higher level randomized control trials, and the treatment benefits were supported by findings in lower-level studies.(Filardo, Di Matteo, Kon, Merli, \& Marcacci, 2016) At this point in time, there is support for use of PRP in the treatment of patellar tendinosis with positive benefit, but questions on type of preparation and number of injections remain unclarified.

\subsection{Achilles Tendinosis}

Use of PRP in the treatment of Achilles tendinosis has been disappointing. de Vos and associates performed a randomized control trial comparing treatment for Achilles tendinosis with a double-blind injection of leukocyte-rich PRP vs saline.(de Vos et al., 2010) Both groups improved and there was no statistically significant difference between the control and PRP groups. This group performed a follow-up study with ultrasound and clinical outcomes at 1 year out from treatment, and again found no difference between the PRP and control groups.(de Jonge et al., 2011) 24 studies were included 
Medical Research Archives, Vol. 5, Issue 6, June 2017

Platelet Rich Plasma: Current Indications and Use in Orthopaedic Care

in a systematic review which demonstrated mixed outcomes, and with higher level studies demonstrating no measurable difference between the PRP and control groups.(Filardo et al., 2016) This collection of studies also suffers from inappropriate comparison as different preparations and numbers of injections were used.

\section{Muscle injury}

Treatment of muscle injury has been proposed to potentially benefit from PRP therapy. While regeneration of muscle tissue is possible after fetal development, many intra-muscular injuries heal with the development of a dense fibrotic scar, which forms at 7-14 days after injury. Therefore, an important aspect that has yet to be ascertained is the timing and appropriate dosing of growth factors to help modulate this process. There is also appropriate concern that the application of PRP may in fact promote the formation of fibrosis and scar due to the high concentrations of growth factors contained in PRP.(Kon et al., 2011) Another factor limiting use of PRP in muscle injury is that the World Anti-Doping Agency (WADA) has listed PRP intramuscular injections as a prohibited intervention for athletes, due to the possible systemic anabolic effects of PRP, making this therapy for muscle injury limited to sports not regulated by WADA.(Kon et al., 2011; Wasterlain, Braun, Harris, Kim, \& Dragoo, 2013)

\subsection{Hamstring Injury}

Hamstring injuries are one of the most common injuries encountered in sports medicine. Despite the high number of injuries, treatment is restricted to physical therapy and conservative measures. Time away from sports can typically be 6 weeks, and risk of recurrent injury persists during the first season following recovery. Initial laboratory data supported the possible use of PRP in shortening the length of time for return to sports and normal function.(Hammond, Hinton, Curl, Muriel, \& Lovering, 2009) Mejia and Bradley treated 12 NFL players with hamstring injuries using single spin PRP (ACP) with the $1^{\text {st }}$ injection $24-48$ hours after injury, and a $2^{\text {nd }}$ injection 5-7 days later, with a $3^{\text {rd }}$ injection administered only if necessary. In their small case series, they noted a 1 day earlier return to play (RTP) for grade I injuries, 5 day earlier RTP for grade II injuries, and a 1 game difference in return to play. (Mejias \& Bradley, 2011) The most notable finding was a reduction in recurrent injury to 0 , where they typically experience 2-4 recurrent hamstring injuries per season over their 20 year experience. These findings have not been validated in other studies. Rettig and colleagues studied the use of 1 injection of leukocyte-rich PRP administered 24-48 hours of injury in NFL players with hamstring injury, but noted no difference in time to return to play between those treated with PRP and those without treatment.(Rettig, Meyer, \& Bhadra, 2013) In the Dutch-HIT study, there was no difference in return to play or difference in 1 year re-injury rate in their double-blind randomized control trial using 2 injections of the same type of single-spin leukocytepoor PRP (ACP) used in Mejia and Bradley's study.(Reurink et al., 2015) The difference between these two studies is that the latter study initiated the $1^{\text {st }}$ injection within 5 days after injury, while Mejia and Bradley administered the first injection 2448 hours after injury. Hamid et al. in a randomized control trial conducted in Malaysia used a single $3 \mathrm{~mL}$ leukocyte-rich PRP injection given between 1-7 days after injury and compared to a physical rehabilitation group.(Hamid, Yusof, \& Mohamed Ali, 2014) The PRP group demonstrated a significantly faster return to sport than the control group with lower pain 
Medical Research Archives, Vol. 5, Issue 6, June 2017

Platelet Rich Plasma: Current Indications and Use in Orthopaedic Care

scores, and concluded PRP therapy to be helpful in early return to sports. However, another 3-arm study conducted in Qatar evaluating soccer football athletes found no difference in RTP when comparing the same leukocyte-rich PRP preparation used in the Hamid et al. study combined with physical rehabilitation when compared to the physical rehabilitation-only group.(Hamilton et al., 2015) Davenport et al. placed a single injection of PRP vs whole blood and found no significant differences in outcomes between the groups.(Davenport et al., 2015) Zanon and colleagues from Italy performed a leukocyte-rich series of 2 or 3 PRP injections starting 24-48 hours after injury in professional soccer football players, and found PRP did not shorten return to sport, had a $12 \%$ recurrent injury rate, but did lead to a smaller scar at the injury site on followup imaging studies.(Zanon et al., 2016) A recent meta-analysis showed no effect of PRP therapy on acute hamstring injury, and identified improved study design for future research efforts.(Pas et al., 2015) The concern with PRP for hamstring muscle injury therapy is confounded by not only preparation variation and number of injections, but also timing for initiation of treatment before stronger recommendations can be made regarding the use of PRP for treatment of acute hamstring injury.

\section{Surgical Augmentation}

Use of PRP therapy for surgical augmentation has initially demonstrated promise in pre-clinical studies, but to date, there has been no conclusive data to suggest efficacy or benefit in terms of clinical outcomes.(Robins, 2014) Two main areas of focus have been in anterior cruciate ligament reconstruction (ACL) surgery and rotator cuff repair.

\subsection{Anterior Cruciate Ligament Reconstruction}

Multiple studies have evaluated the effects of PRP on ACL graft maturation which would presume better healing and lower re-tear rates. A recent systematic review concluded that PRP may provide a $20-30 \%$ beneficial effect on graft maturation, but that there is no evidence of improved graft-bone interface healing, nor is there any difference in clinical outcomes.(Vavken, Sadoghi, \& Murray, 2011) In both a level I randomized control trial, and in the large Multicenter Orthopaedic Outcomes Network (MOON) ACL study cohort, addition of PRP to ACL allografts was shown to have no significant difference in any outcomes or parameters following surgery.(Magnusson, Romano, Hallberg, Wadenvik, \& Breimer, 1998; Nin, Gasque, Azcarate, Beola, \& Gonzalez, 2009) In relation to treatment of patellar tendinopathy, two studies have evaluated adding 2 different types of PRP gel preparations to the harvest site of the central portion of the patellar tendon created during ACL reconstruction.(de Almeida et al., 2012; Seijas et al., 2013) Both studies showed significant reduction in early postoperative pain, and de Almeida et al. demonstrated significantly-improved healing compared to the control group at 6 months on MRI. However, one of the limitations to de Almedia et al.'s application of PRP is that $50 \mathrm{~mL}$ of PRP was applied to the harvest site, which requires $450 \mathrm{~mL}$ of blood volume be at least temporarily withdrawn for preparation of this large volume of PRP. In Sejia and colleagues study, only 4cc of PRP (PRGF) was applied to the donor sites, which reduced pain during the first 2 months compared to the control group. While use of PRP may reduce pain in the postoperative period, further research is needed to determine if the 
cost-effectiveness of this application of PRP is a reasonable option.

\subsection{Rotator Cuff Repair}

PRP applied to augment rotator cuff repair has followed the pattern of early promising results in pre-clinical and laboratory data, but without demonstrating benefit with application in clinical series.(Robins, 2014) In a randomized control trial, application of PRP fibrin matrix (PRFM) to the rotator cuff repair site was found to correlate with worse healing rates, indicating the possibility the PRP gel interfered rather than benefited tendon-tobone healing.(Rodeo et al., 2012) Out of 18 randomized control trials on PRP augmentation in rotator cuff repair, 10 demonstrated no differences between intervention and control groups, 2 demonstrated improved pain control during the $1^{\text {st }}$ two months after surgery, and 5 showed lower re-tear rates on imaging but not necessarily any difference in clinical outcomes.(Filardo et al., 2016) The high degree of heterogeneity in application and types of injections utilized in rotator cuff repair make interpreting which type of PRP and when to inject difficult to determine. In addition the cost-effectiveness of adding PRP to rotator cuff repair would have to show at least a reduced re-tear rate of $9.1 \%$ to be worth adding to surgical treatment.(Samuelson, Odum, \& Fleischli, 2016) Metanalysis demonstrated PRP had no effect on large rotator cuff repairs, did help in small and medium tears at reducing re-tear rate, but was too costly to make adding PRP to this cohort of injury justifiable.(Vavken et al., 2015)

\section{Conclusion}

PRP, as a biologic therapy, presents the possibility of benefit to multiple musculoskeletal injury sites. Use of leukocyte-poor PRP in the treatment of knee osteoarthritis has demonstrated significant benefits in multiple trials and case series. Use of PRP in the treatment of lateral epicondylitis, in particular with leukocyterich preparations, had some evidence to support its use for therapeutic benefit. A small amount of evidence supports the possibility of PRP helping in treating partial UCL tears in the elbow. There is also limited evidence to consider the use of PRP in the management of patellar tendinosis. Most evidence supports not using PRP in the treatment of Achilles tendon partial tears or tendinopathy. Use of PRP in muscle injury and specifically in hamstring injury has mixed results in the data, and it appears that besides type and number of injections, the timing of PRP injections is important in promoting muscle regeneration versus inadvertently stimulating increased fibrosis across the injury site. Finally, PRP in augmenting $\mathrm{ACL}$ and rotator cuff tendon repair have mixed results and cannot be recommended for routine use at this point in time. 
Medical Research Archives, Vol. 5, Issue 6, June 2017

Platelet Rich Plasma: Current Indications and Use in Orthopaedic Care

\section{References}

1. Ahmad, Z., Brooks, R., Kang, S. N., Weaver, H., Nunney, I., TytherleighStrong, G., \& Rushton, N. (2013). The effect of platelet-rich plasma on clinical outcomes in lateral epicondylitis. Arthroscopy, 29(11), 1851-1862. doi:10.1016/j.arthro.2013.07.272

2. Anitua, E., Sanchez, M., Nurden, A. T., Nurden, P., Orive, G., \& Andia, I. (2006). New insights into and novel applications for platelet-rich fibrin therapies. Trends Biotechnol, 24(5), 227-234.

doi:10.1016/j.tibtech.2006.02.010

3. Braun, H. J., Kim, H. J., Chu, C. R., \& Dragoo, J. L. (2014). The effect of platelet-rich plasma formulations and blood products on human synoviocytes: implications for intraarticular injury and therapy. Am J Sports Med, 42(5), 1204-1210. doi:10.1177/0363546514525593

4. Cerza, F., Carni, S., Carcangiu, A., Di Vavo, I., Schiavilla, V., Pecora, A., . . . Ciuffreda, M. (2012). Comparison between hyaluronic acid and plateletrich plasma, intra-articular infiltration in the treatment of gonarthrosis. Am J Sports Med, 40(12), 2822-2827. doi:10.1177/0363546512461902

5. Charousset, C., Zaoui, A., Bellaiche, L., \& Bouyer, B. (2014). Are multiple platelet-rich plasma injections useful for treatment of chronic patellar tendinopathy in athletes? a prospective study. Am J Sports Med, 42(4), 906911. doi:10.1177/0363546513519964

6. Cole, B. J., Karas, V., Hussey, K., Pilz, K., \& Fortier, L. A. (2017). Hyaluronic Acid Versus Platelet-Rich
Plasma: A Prospective, Double-Blind Randomized Controlled Trial Comparing Clinical Outcomes and Effects on Intra-articular Biology for the Treatment of Knee Osteoarthritis. Am J Sports Med, 45(2), 339-346. doi:10.1177/0363546516665809

7. Creaney, L., \& Hamilton, B. (2008). Growth factor delivery methods in the management of sports injuries: the state of play. Br J Sports Med, 42(5), 314-320. doi:10.1136/bjsm.2007.040071

8. Dai, W. L., Zhou, A. G., Zhang, H., \& Zhang, J. (2017). Efficacy of PlateletRich Plasma in the Treatment of Knee Osteoarthritis: A Meta-analysis of Randomized Controlled Trials. Arthroscopy, 33(3), 659-670 e651. doi:10.1016/j.arthro.2016.09.024

9. Davenport, K. L., Campos, J. S., Nguyen, J., Saboeiro, G., Adler, R. S., \& Moley, P. J. (2015). UltrasoundGuided Intratendinous Injections With Platelet-Rich Plasma or Autologous Whole Blood for Treatment of Proximal Hamstring Tendinopathy: A Double-Blind Randomized Controlled Trial. J Ultrasound Med, 34(8), 14551463. doi:10.7863/ultra.34.8.1455

10. de Almeida, A. M., Demange, M. K., Sobrado, M. F., Rodrigues, M. B., Pedrinelli, A., \& Hernandez, A. J. (2012). Patellar tendon healing with platelet-rich plasma: a prospective randomized controlled trial. Am J Sports Med, 40(6), 1282-1288. doi:10.1177/0363546512441344

11. de Jonge, S., de Vos, R. J., Weir, A., van Schie, H. T., Bierma-Zeinstra, S. M., Verhaar, J. A., . . . Tol, J. L. (2011). 
One-year follow-up of platelet-rich plasma treatment in chronic Achilles tendinopathy: a double-blind randomized placebo-controlled trial. Am J Sports Med, 39(8), 1623-1629. doi:10.1177/0363546511404877

12. de Vos, R. J., Weir, A., van Schie, H. T., Bierma-Zeinstra, S. M., Verhaar, J. A., Weinans, H., \& Tol, J. L. (2010). Platelet-rich plasma injection for chronic Achilles tendinopathy: a randomized controlled trial. JAMA, 303(2), doi:10.1001/jama.2009.1986

13. de Vos, R. J., Windt, J., \& Weir, A. (2014). Strong evidence against platelet-rich plasma injections for chronic lateral epicondylar tendinopathy: a systematic review. $\mathrm{Br}$ $J$ Sports Med, 48(12), 952-956. doi:10.1136/bjsports-2013-093281

14. DeLong, J. M., Russell, R. P., \& Mazzocca, A. D. (2012). Platelet-rich plasma: the PAW classification system. Arthroscopy, 28(7), 998-1009. doi:10.1016/j.arthro.2012.04.148

15. Dines, J. S., Williams, P. N., ElAttrache, N., Conte, S., Tomczyk, T., Osbahr, D. C., . . . Ahmad, C. S. (2016). Platelet-Rich Plasma Can Be Used to Successfully Treat Elbow Ulnar Collateral Ligament Insufficiency in High-Level Throwers. Am J Orthop (Belle Mead NJ), 45(5), 296-300.

16. Dragoo, J. L., Wasterlain, A. S., Braun, H. J., \& Nead, K. T. (2014). Plateletrich plasma as a treatment for patellar tendinopathy: a double-blind, randomized controlled trial. Am $J$ Sports Med, 42(3), 610-618. doi:10.1177/0363546513518416

17. Durant, T. J., Dwyer, C. R., McCarthy, M. B., Cote, M. P., Bradley, J. P., \&
Mazzocca, A. D. (2017). Protective Nature of Platelet-Rich Plasma Against Chondrocyte Death When Combined With Corticosteroids or Local Anesthetics. Am J Sports Med, 45(1), 218-225. doi:10.1177/0363546516664161

18. Filardo, G., Di Matteo, B., Kon, E., Dhillon, M. S., Patel, S., \& Marwaha, N. (2013). Platelet-rich plasma for knee osteoarthritis. Am J Sports Med, 41(9), NP42-43. doi:10.1177/0363546513502635

19. Filardo, G., Di Matteo, B., Kon, E., Merli, G., \& Marcacci, M. (2016). Platelet-rich plasma in tendon-related disorders: results and indications. Knee Surg Sports Traumatol Arthrosc. doi:10.1007/s00167-016-4261-4

20. Filardo, G., Kon, E., Buda, R., Timoncini, A., Di Martino, A., Cenacchi, A., . . . Marcacci, M. (2011). Platelet-rich plasma intraarticular knee injections for the treatment of degenerative cartilage lesions and osteoarthritis. Knee Surg Sports Traumatol Arthrosc, 19(4), 528-535. doi:10.1007/s00167-010$1238-6$

21. Filardo, G., Kon, E., Della Villa, S., Vincentelli, F., Fornasari, P. M., \& Marcacci, M. (2010). Use of plateletrich plasma for the treatment of refractory jumper's knee. Int Orthop, 34(6), 909-915. doi:10.1007/s00264009-0845-7

22. Filardo, G., Kon, E., Pereira Ruiz, M. T., Vaccaro, F., Guitaldi, R., Di Martino, A., . . . Marcacci, M. (2012). Plateletrich plasma intra-articular injections for cartilage degeneration and osteoarthritis: single- versus doublespinning approach. Knee Surg Sports 
Traumatol Arthrosc, 20(10), 20822091. doi:10.1007/s00167-011-1837-X

23. Filardo, G., Kon, E., Roffi, A., Di Matteo, B., Merli, M. L., \& Marcacci, M. (2015). Platelet-rich plasma: why intra-articular? A systematic review of preclinical studies and clinical evidence on PRP for joint degeneration. Knee Surg Sports Traumatol Arthrosc, 23(9), 24592474. doi:10.1007/s00167-013-2743-1

24. Fitzpatrick, J., Bulsara, M., \& Zheng, M. H. (2017). The Effectiveness of Platelet-Rich Plasma in the Treatment of Tendinopathy. Am J Sports Med, 45(1), 226-233. doi:10.1177/0363546516643716

25. Fitzpatrick, J., Bulsara, M. K., McCrory, P. R., Richardson, M. D., \& Zheng, M. H. (2017). Analysis of Platelet-Rich Plasma Extraction: Variations in Platelet and Blood Components Between 4 Common Commercial Kits. Orthop J Sports Med, 5(1), 2325967116675272. doi: $10.1177 / 2325967116675272$

26. Gosens, T., Den Oudsten, B. L., Fievez, E., van 't Spijker, P., \& Fievez, A. (2012). Pain and activity levels before and after platelet-rich plasma injection treatment of patellar tendinopathy: a prospective cohort study and the influence of previous treatments. Int Orthop, $\quad 36(9), \quad$ 1941-1946. doi:10.1007/s00264-012-1540-7

27. Gosens, T., \& Mishra, A. K. (2014). Editorial in response to the systematic review by de Vos et al: 'Strong evidence against platelet-rich plasma injections for chronic lateral epicondylar tendinopathy: a systematic review'. Br J Sports Med, 48(12), 945-946. doi:10.1136/bjsports2014-093704
28. Gosens, T., Peerbooms, J. C., van Laar, W., \& den Oudsten, B. L. (2011). Ongoing positive effect of platelet-rich plasma versus corticosteroid injection in lateral epicondylitis: a double-blind randomized controlled trial with 2year follow-up. Am J Sports Med, 39(6), 1200-1208. doi:10.1177/0363546510397173

29. Hall, M. P., Band, P. A., Meislin, R. J., Jazrawi, L. M., \& Cardone, D. A. (2009). Platelet-rich plasma: current concepts and application in sports medicine. J Am Acad Orthop Surg, 17(10), 602-608.

30. Hamid, M. S., Yusof, A., \& Mohamed Ali, M. R. (2014). Platelet-rich plasma (PRP) for acute muscle injury: a systematic review. PLoS One, 9(2), e90538.

doi:10.1371/journal.pone.0090538

31. Hamilton, B., Tol, J. L., Almusa, E., Boukarroum, S., Eirale, C., Farooq, A., . . . Chalabi, H. (2015). Plateletrich plasma does not enhance return to play in hamstring injuries: a randomised controlled trial. $\mathrm{Br} J$ Sports Med, 49(14), 943-950. doi:10.1136/bjsports-2015-094603

32. Hammond, J. W., Hinton, R. Y., Curl, L. A., Muriel, J. M., \& Lovering, R. M. (2009). Use of autologous platelet-rich plasma to treat muscle strain injuries. Am J Sports Med, 37(6), 1135-1142. doi:10.1177/0363546508330974

33. Hsu, W. K., Mishra, A., Rodeo, S. R., Fu, F., Terry, M. A., Randelli, P., . . . Kelly, F. B. (2013). Platelet-rich plasma in orthopaedic applications: evidence-based recommendations for treatment. J Am Acad Orthop Surg, 21(12), 739-748. doi:10.5435/JAAOS21-12-739 
34. Hunt, T. J. (2017). Editorial Commentary: The Time Has Come to Try Intra-articular Platelet-Rich Plasma Injections for Your Patients With Symptomatic Knee Osteoarthritis. Arthroscopy, 33(3), 671-672.

doi:10.1016/j.arthro.2016.12.006

35. Kon, E., Filardo, G., Delcogliano, M., Presti, M. L., Russo, A., Bondi, A., ... Marcacci, M. (2009). Platelet-rich plasma: new clinical application: a pilot study for treatment of jumper's knee. Injury, 40(6), 598-603. doi:10.1016/j.injury.2008.11.026

36. Kon, E., Filardo, G., Di Martino, A., \& Marcacci, M. (2011). Platelet-rich plasma (PRP) to treat sports injuries: evidence to support its use. Knee Surg Sports Traumatol Arthrosc, 19(4), 516-527. doi:10.1007/s00167-0101306-y

37. Lebiedzinski, R., Synder, M., Buchcic, P., Polguj, M., Grzegorzewski, A., \& Sibinski, M. (2015). A randomized study of autologous conditioned plasma and steroid injections in the treatment of lateral epicondylitis. Int Orthop, 39(11), 2199-2203. doi:10.1007/s00264-015-2861-0

38. Magnusson, S., Romano, E. L., Hallberg, E., Wadenvik, H., \& Breimer, M. E. (1998). Soluble saccharides block the inhibition of agonist-induced human platelet aggregation observed after in vitro incubation of human platelet-rich plasma with porcine aortic endothelial cells. Transpl Int, 11(5), 345-352.

39. Marx, R. E. (2004). Platelet-rich plasma: evidence to support its use. J Oral Maxillofac Surg, 62(4), 489-496.
40. Mazzocca, A. D., McCarthy, M. B., Chowaniec, D. M., Cote, M. P., Romeo, A. A., Bradley, J. P., . . . Beitzel, K. (2012). Platelet-rich plasma differs according to preparation method and human variability. $J$ Bone Joint Surg Am, 94(4), 308-316. doi:10.2106/JBJS.K.00430

41. Meheux, C. J., McCulloch, P. C., Lintner, D. M., Varner, K. E., \& Harris, J. D. (2016). Efficacy of Intraarticular Platelet-Rich Plasma Injections in Knee Osteoarthritis: A Systematic Review. Arthroscopy, 32(3), 495-505.

doi:10.1016/j.arthro.2015.08.005

42. Mejias, H. A., \& Bradley, J. P. (2011). The Effects of Platelet-Rich Plasma on Muscle: Basic Science and Clinical Application. Operative Techniques in Sports Medicine, 19(3), 149-153.

43. Mishra, A., Harmon, K., Woodall, J., \& Vieira, A. (2012). Sports medicine applications of platelet rich plasma. Curr Pharm Biotechnol, 13(7), 11851195.

44. Mishra, A., \& Pavelko, T. (2006). Treatment of chronic elbow tendinosis with buffered platelet-rich plasma. Am J Sports Med, 34(11), 1774-1778. doi: $10.1177 / 0363546506288850$

45. Mishra, A. K., Skrepnik, N. V., Edwards, S. G., Jones, G. L., Sampson, S., Vermillion, D. A., ... Rettig, A. C. (2014). Efficacy of platelet-rich plasma for chronic tennis elbow: a double-blind, prospective, multicenter, randomized controlled trial of 230 patients. Am J Sports Med, 42(2), 463-471. doi: $10.1177 / 0363546513494359$ 
46. Nin, J. R., Gasque, G. M., Azcarate, A. V., Beola, J. D., \& Gonzalez, M. H. (2009). Has platelet-rich plasma any role in anterior cruciate ligament allograft healing? Arthroscopy, 25(11), 1206-1213. doi:10.1016/j.arthro.2009.06.002

47. Osterman, C., McCarthy, M. B., Cote, M. P., Beitzel, K., Bradley, J., Polkowski, G., \& Mazzocca, A. D. (2015). Platelet-Rich Plasma Increases Anti-inflammatory Markers in a Human Coculture Model for Osteoarthritis. Am J Sports Med, 43(6), 1474-1484. doi:10.1177/0363546515570463

48. Pas, H. I., Reurink, G., Tol, J. L., Weir, A., Winters, M., \& Moen, M. H. (2015). Efficacy of rehabilitation (lengthening) exercises, platelet-rich plasma injections, and other conservative interventions in acute hamstring injuries: an updated systematic review and meta-analysis. Br J Sports Med, 49(18), 1197-1205. doi:10.1136/bjsports-2015-094879

49. Peerbooms, J. C., Sluimer, J., Bruijn, D. J., \& Gosens, T. (2010). Positive effect of an autologous platelet concentrate in lateral epicondylitis in a double-blind randomized controlled trial: platelet-rich plasma versus corticosteroid injection with a 1-year follow-up. Am J Sports Med, 38(2), 255-262.

doi:10.1177/0363546509355445

50. Podesta, L., Crow, S. A., Volkmer, D., Bert, T., \& Yocum, L. A. (2013). Treatment of partial ulnar collateral ligament tears in the elbow with platelet-rich plasma. Am J Sports Med, 41(7), 1689-1694. doi: $10.1177 / 0363546513487979$
51. Rettig, A. C., Meyer, S., \& Bhadra, A. K. (2013). Platelet-Rich Plasma in Addition to Rehabilitation for Acute Hamstring Injuries in NFL Players: Clinical Effects and Time to Return to Play. Orthop J Sports Med, 1(1), 2325967113494354. doi:10.1177/2325967113494354

52. Reurink, G., Goudswaard, G. J., Moen, M. H., Weir, A., Verhaar, J. A., Bierma-Zeinstra, S. M., . . . Dutch, H. I. T. s. I. (2015). Rationale, secondary outcome scores and 1-year follow-up of a randomised trial of platelet-rich plasma injections in acute hamstring muscle injury: the Dutch Hamstring Injection Therapy study. $\mathrm{Br} J$ Sports Med, 49(18), 1206-1212. doi:10.1136/bjsports-2014-094250

53. Riboh, J. C., Saltzman, B. M., Yanke, A. B., Fortier, L., \& Cole, B. J. (2016). Effect of Leukocyte Concentration on the Efficacy of Platelet-Rich Plasma in the Treatment of Knee Osteoarthritis. Am J Sports Med, 44(3), 792-800. doi:10.1177/0363546515580787

54. Robins, R. J. (2014). PRP in ACL reconstruction--another indication for surgical augmentation? J Surg Res, 186(1), 105-106. doi:10.1016/j.jss.2013.02.013

55. Rodeo, S. A., Delos, D., Williams, R. J., Adler, R. S., Pearle, A., \& Warren, R. F. (2012). The effect of platelet-rich fibrin matrix on rotator cuff tendon healing: a prospective, randomized clinical study. Am J Sports Med, 40(6), 1234-1241. doi:10.1177/0363546512442924

56. Sampson, S., Gerhardt, M., \& Mandelbaum, B. (2008). Platelet rich plasma injection grafts for musculoskeletal injuries: a review. Curr Rev Musculoskelet Med, 1(3-4), 
165-174. doi:10.1007/s12178-008$9032-5$

57. Samuelson, E. M., Odum, S. M., \& Fleischli, J. E. (2016). The CostEffectiveness of Using Platelet-Rich Plasma During Rotator Cuff Repair: A Markov Model Analysis. Arthroscopy, 32(7), 1237-1244. doi:10.1016/j.arthro.2015.12.018

58. Seijas, R., Ares, O., Catala, J., AlvarezDiaz, P., Cusco, X., \& Cugat, R. (2013). Magnetic resonance imaging evaluation of patellar tendon graft remodelling after anterior cruciate ligament reconstruction with or without platelet-rich plasma. $J$ Orthop Surg (Hong Kong), 21(1), 10-14. doi:10.1177/230949901302100105

59. Smith, P. A. (2016). Intra-articular Autologous Conditioned Plasma Injections Provide Safe and Efficacious Treatment for Knee Osteoarthritis: An FDA-Sanctioned, Randomized, Double-blind, Placebocontrolled Clinical Trial. Am J Sports Med, 44(4), 884-891. doi: $10.1177 / 0363546515624678$

60. Vavken, P., Sadoghi, P., \& Murray, M. M. (2011). The effect of platelet concentrates on graft maturation and graft-bone interface healing in anterior cruciate ligament reconstruction in human patients: a systematic review of controlled trials. Arthroscopy, 27(11), 1573-1583.

doi:10.1016/j.arthro.2011.06.003

61. Vavken, P., Sadoghi, P., Palmer, M., Rosso, C., Mueller, A. M., Szoelloesy, G., \& Valderrabano, V. (2015). Platelet-Rich Plasma Reduces Retear Rates After Arthroscopic Repair of Small- and Medium-Sized Rotator Cuff Tears but Is Not Cost-Effective. Am J Sports Med, 43(12), 3071-3076. doi:10.1177/0363546515572777

62. Wasterlain, A. S., Braun, H. J., Harris, A. H., Kim, H. J., \& Dragoo, J. L. (2013). The systemic effects of platelet-rich plasma injection. Am J Sports Med, 4l(1), 186-193. doi:10.1177/0363546512466383

63. Williams, P. N., Moran, G., Bradley, J. P., N, S. E., \& Dines, J. S. (2015). Platelet-rich plasma and other cellular strategies in orthopedic surgery. Curr Rev Musculoskelet Med, 8(1), 32-39. doi:10.1007/s12178-014-9246-7

64. Zanon, G., Combi, F., Combi, A., Perticarini, L., Sammarchi, L., \& Benazzo, F. (2016). Platelet-rich plasma in the treatment of acute hamstring injuries in professional football players. Joints, 4(1), 17-23. doi:10.11138/jts/2016.4.1.017

\section{Funding Source}

Financial support for this review has been provided from research funds of the $10^{\text {th }}$ Surgical Operations Squadron, $10^{\text {th }}$ Medical Group, United States Air Force Academy, Colorado.

\section{Disclaimer}

*The viewpoints presented in this article do not represent the official position of the United States government, the Department of Defense, the United States Air Force, or the United States Air Force Academy. 